\title{
SIMULATIONS OF A GAS-FILLED HELICAL MUON BEAM COOLING CHANNEL*
}

\author{
Katsuya Yonehara, Daniel Kaplan, Illinois Institute of Technology, Chicago, Illinois, U.S.A. \\ Kevin Beard, S. Alex Bogacz, Yaroslav Derbenev, Jefferson Lab, Newport News, Virginia, U.S.A. \\ Rolland Johnson, Kevin Paul, Thomas Roberts, Muons, Inc., Batavia, Illinois, U.S.A.
}

\begin{abstract}
A helical cooling channel (HCC) has been proposed to quickly reduce the six dimensional phase space of muon beams for muon colliders, neutrino factories, and intense muon sources. The HCC is composed of solenoidal, helical dipole, and helical quadrupole current coils to provide focusing and dispersion needed for emittance exchange as the beam follows an equilibrium helical orbit. Inside the coils constituting the HCC examined here, a series of RF cavities filled with dense hydrogen gas acts as the energy absorber for ionization cooling and also suppressed RF breakdown. Two Monte Carlo simulation programs have been developed to compare HCC performance with analytic predictions and to begin the process of optimizing practical designs that could be built in the near future. We discuss the programs, the comparisons with the analytical theory, and the prospects for a HCC design with the capability to reduce the sixdimensional phase space emittance of a muon beam by a factor of over five orders of magnitude in a linear channel less than 100 meters long.
\end{abstract}

\section{INTRODUCTION}

Helical magnets have been used for some time in the control of spin precession in polarized beam devices. One advantage of helical magnets is that, in conjunction with a solenoid field, the fields can be continually focusing and dispersive. Continually focusing, dispersive fields are necessary for the simultaneous transverse and longitudinal ionization cooling of muon beams.

High-pressure, hydrogen gas-filled RF cavities have been proposed to provide large gradient acceleration of muon beams. The high-pressure gas serves to suppress $\mathrm{RF}$ breakdown and also serves as the muon ionization cooling material. The peak RF gradient and RF phase are chosen to compensate for energy loss in the gas, maintaining the design reference energy down the channel. We believe that the suppression of RF breakdown by the gas will also allow operation of the cavities inside strong magnetic fields.

This article shows the cooling results of simulations for such an HCC in two different simulation software packages designed for accurate modeling of muon ionization: ICOOL and G4Beamline.

\section{SIMULATION SOFTWARE}

Only a handful of simulation codes exist that can properly handle beam interactions with matter and particle decay, both of which are necessary for proper simulation of muon cooling and muon beams in general. Two such codes are ICOOL and G4Beamline.

\section{ICOOL}

ICOOL is a Monte Carlo beam tracking package, written in Fortran 77, borrowing routines to handle particle interactions with matter and decay from GEANT3. It is developed and maintained by R. Fernow [2] at Brookhaven National Laboratory specifically for development of muon colliders and neutrino factories.

\section{G4Beamline}

G4Beamline is a Monte Carlo beamline simulation package built on top of the GEANT4 toolkit [3] in $\mathrm{C}++$. It is designed to have all of the particle interaction, tracking, and 3D geometry capabilities of the GEANT4 toolkit and the fully 3D graphics capability of the OpenInventor libraries, all within the framework of a beamline design program. It is developed and maintained by T. Roberts of Muons, Inc. [4].

\section{SIMULATION RESULTS}

HCC dynamics and beam cooling performance have been examined in some detail using descriptions of magnetic fields that satisfy Maxwell's equations [1]. These field descriptions agree well with the measured fields from real helical magnets, such as the AGS snake [5]. Routines for computing the analytically determined fields have been written into both ICOOL and G4Beamline for simulation. For simplicity, we have started this simulation effort with a reference muon beam momentum of $250 \mathrm{MeV} / \mathrm{c}$, a reference orbit helical pitch angle of 45 degrees (helical pitch, $\kappa=\tan 45^{\circ}=1$ ), and a helical period of 1 meter $(\lambda=1 \mathrm{~m})$. We have then demanded that the three cooling decrements (two transverse and one longitudinal) be equal. Under these specifications, the fields for the solenoid, helical dipole, and helical quadrupole are uniquely determined [1], listed in Table 1 .

*Work supported in part by DOE STTR/SBIR grants DE-FG0202ER86145 and 03ER83722. 
Table 1: HCC design parameters. Helical Pitch, $\kappa$, is defined as tangent of the helical pitch angle of the reference orbit. Helical field strengths are quoted at the radius of the helical reference orbit, a.

\begin{tabular}{|l|l|l|}
\hline Ref. Orbit Momentum: & $\mathbf{p}$ & $250 \mathrm{MeV} / \mathrm{c}$ \\
\hline Helical Ref. Orbit Pitch: & $\mathbf{\kappa}$ & 1 \\
\hline Helical Period: & $\lambda$ & $1 \mathrm{~m}$ \\
\hline Helical Ref. Orbit Radius: & $\mathbf{a}$ & $0.159 \mathrm{~m}$ \\
\hline Solenoid Field Strength: & $\mathbf{B}$ & $6.99 \mathrm{~T}$ \\
\hline Helical Dipole Strength: & $\mathbf{b}$ & $1.64 \mathrm{~T}$ \\
\hline Helical Quadrupole Strength: & $\mathbf{b}^{\prime}$ & $-0.879 \mathrm{~T} / \mathrm{m}$ \\
\hline Helical Single-Period & $\mathbf{Q}_{+}$ & 0.913 \\
Transverse Tunes: & $\mathbf{Q}_{-}$ & 0.749 \\
\hline Transition Momentum & $\mathbf{p}_{\mathbf{t}}$ & $60.2 \mathrm{MeV} / \mathrm{c}$ \\
\hline RF Frequency: & $\mathbf{f}_{\mathbf{R F}}$ & $200 \mathrm{MHz}$ \\
\hline RF Phase: & $\phi_{\mathbf{R F}}$ & $140^{\circ}$ \\
\hline RF Gradient: & $\mathbf{E}_{\mathbf{R F}}$ & $31 \mathrm{MV} / \mathrm{m}$ \\
\hline
\end{tabular}

High-pressure, gaseous hydrogen-filled RF cavities [6] are placed inside the helical magnets. We assume 100 atm gaseous hydrogen at $77 \mathrm{~K}$ inside the cavities, which corresponds to almost half the density of liquid hydrogen. After considering the fact that the particle trajectories are not aligned with the central axis of the cavities (i.e., they are helical), an average RF gradient of $20 \mathrm{MV} / \mathrm{m}$ must be applied to the beam to compensate for energy loss in the gas. Since we are above transition, we select an RF phase of $140^{\circ}$ such that the peak RF gradient of our cavities must be $31 \mathrm{MV} / \mathrm{m}$. The simulations assume sinusoidal time-dependence but no radial dependence in the RF fields. Experiments at Fermilab's Mucool Test Area (MTA) have been proposed to test the operation of cavities under similar conditions [7].
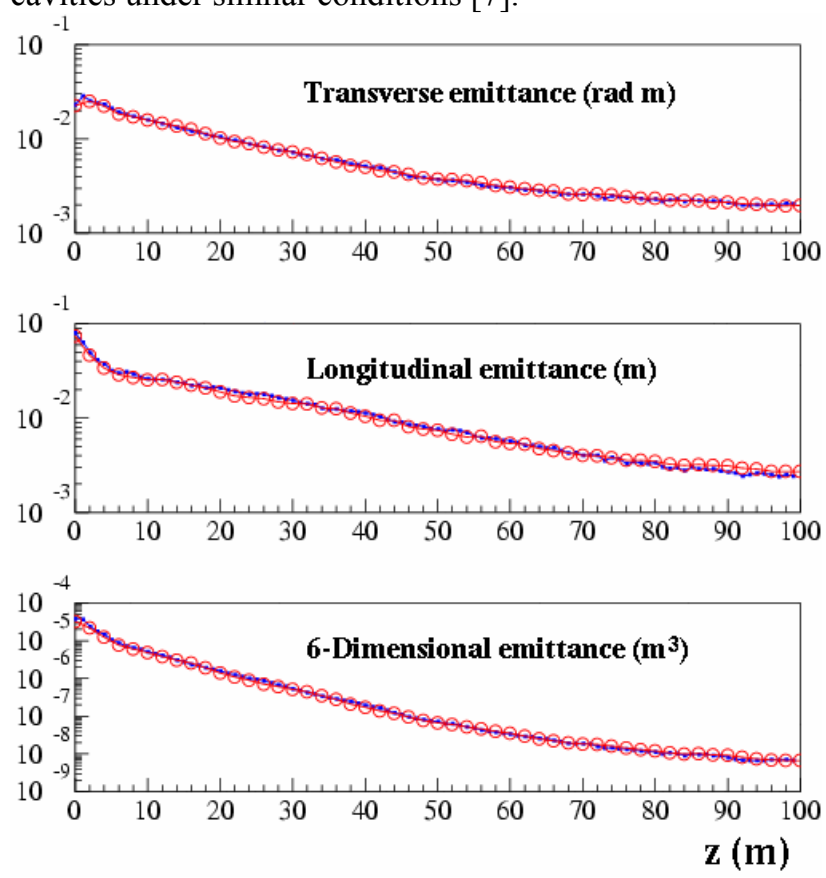

Figure 1: Evolution of emittance over the length of the 100 meter HCC. Results in red are from G4Beamline and blue from ICOOL.
Figure 1 shows the evolution of the normalized transverse, longitudinal, and six-dimensional emittance down the length of the HCC, as calculated by the ICOOL post-processing tool ECALC9 [8]. Perfect matching into the channel is assumed. Results are found by considering only the surviving subset of muons from a large beam that saturates the acceptance of the channel, where survival is determined by whether the muon reaches the end of the channel. We see very good agreement between the two simulation codes. The initial six-dimensional acceptance of the channel is very large, around $40 \mathrm{~cm}^{3}(22 \mathrm{~mm}$ transverse, $80 \mathrm{~mm}$ longitudinal), larger than the initial emittance of the muon beam considered in the Neutrino Factory Feasibility Study 2a. The HCC cools the beam by a factor of approximately 4800 over a channel length of $100 \mathrm{~m}$, down to a six-dimensional emittance of $8 \mathrm{~mm}^{3}$ (2 $\mathrm{mm}$ transverse, $2 \mathrm{~mm}$ longitudinal).
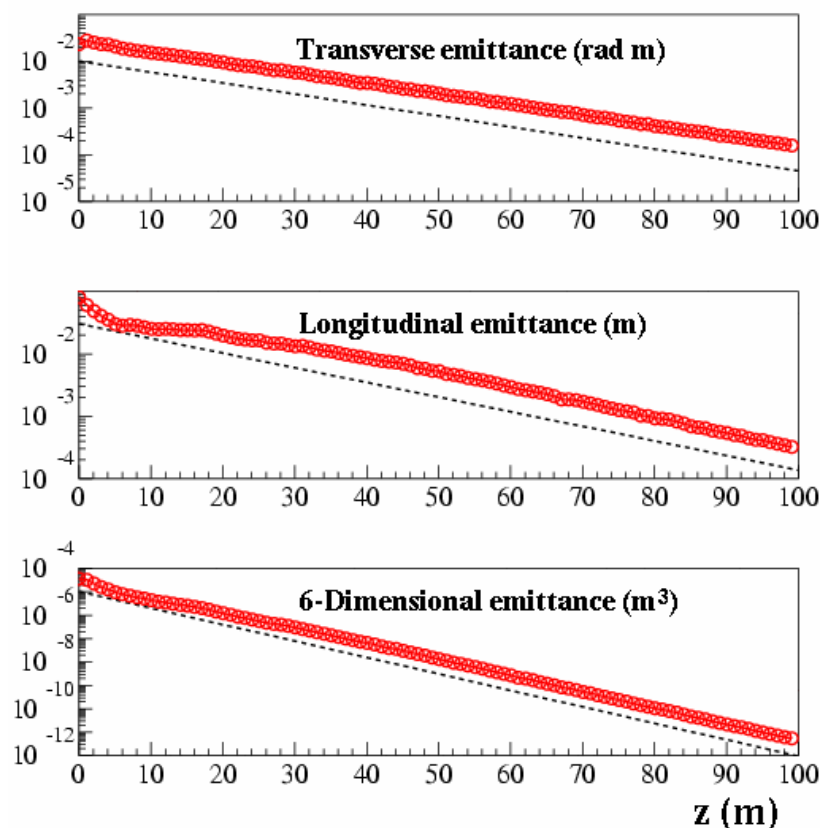

Figure 2: Emittance evolution, as calculated by ICOOL and ECALC9, down the channel with multiple scattering and straggling turned off. The dashed black line shows the predicted slope of the curves, drawn below the simulated data (rather than on top) just to make it easier to see.

Figure 2 shows the evolution of transverse and longitudinal emittance down the channel when multiple scattering and energy straggling is turned off in ICOOL. This demonstrates perfect exponential decay of the emittances, from which we extract the cooling decrements. The predicted cooling decrements are $\Lambda_{\text {trans }}=$ $\Lambda_{\text {long }}=1 / 3 \Lambda_{6 \mathrm{D}}=5.4 \mathrm{~cm}^{-1}$. The fitted cooling decrements are $\Lambda_{\text {trans }}=5.2 \mathrm{~cm}^{-1}$, and $\Lambda_{\text {long }}=5.8 \mathrm{~cm}^{-1}$, and $1 / 3 \Lambda_{6 \mathrm{D}}=5.3$ $\mathrm{cm}^{-1}$. We see good agreement between theory and simulation. 


\section{FUTURE PROSPECTS}

With different design parameter choices, it is, in principle, possible to create a helical cooling channel that has a lower equilibrium emittance and smaller acceptance. Such a helical cooling channel can be used following the channel described in the previous section. To be more efficient, one might consider an HCC equivalent to that described in the previous section but with a shorter length, followed by a series of HCC's with sequentially smaller equilibrium emittances and acceptances. Then, we more efficiently take advantage of the faster cooling rate achieved by a beam that is further from equilibrium. Such a design can maximize cooling and minimize the required length of the channel.

Finalized designs depend on the feasibility of the highpressure, hydrogen gas-filled RF cavities [6] to be determined in future experiments [7]. If the required gradients are not achievable in such high fields, then it may be possible to created a channel that alternates between the gas-filled helical magnets and high-gradient, gas-filled RF. Such a design would not be as efficient, but it could still achieved dramatic cooling. Other complications that may be uncovered during the proposed experiments could require alternative designs, but the design discussed in the prior section would be the most efficient.

\section{REFERENCES}

[1] Six-dimensional muon beam cooling using a homogeneous absorber, Y. S. Derbenev and R. P. Johnson, http://www-mucool.fnal.gov/menotes/ public/pdf/muc0284/muc0284.pdf

[2] http://pubweb.bnl.gov/people/fernow/icool/

[3] http://geant4.web.cern.ch/geant4/

[4] http://www.muonsinc.com/g4beamline.html

[5] Field Measurements In The AGS Warm Snake, J. Takano et al., Proceedings of EPAC 2004, pp 2116

[6] Mark II High-Pressure RF Test Cell Measurement with Molybdenum Electrodes at Lab G, R. E Hartline, R. P. Johnson, M. Kuchnir, C. M. Ankenbrandt, A. Moretti, M. Popovic, D. M. Kaplan, K. Yonehara, http://www-mucool.fnal.gov/monotes/ public/pdf/muc0285/muc0285.pdf

[7] Studies of RF Breakdown of Metals in Dense Gases, P. M. Hanlet et al, this conference

[8] Physics analysis performed by ECALC9, R. Fernow, http://www-mucool.fnal.gov/monotes/ public/pdf/muc0280/muc0280.pdf 